\title{
Cambios químicos y biológicos del suelo provocados por Pteridium aquilinum (L.) Kuhn en áreas de influencia de la reserva de la biosfera de Calakmul, Campeche Chemical and biological soil changes caused by Pteridium aquilinum (L.) Kuhn in areas of influence of the reserve of the biosphere of Calakmul, Campeche
}

\author{
Carlos Valdez-Ramírez ${ }^{1 *}$ (D), Samuel Israel Levy-Tacher ${ }^{1(\mathbb{D})}$, Noé Samuel León-Martínez ${ }^{1}$ (D), \\ Darío Alejandro Navarrete-Gutiérrez ${ }^{1}$ (i) y Ángel Isauro Ortiz-Ceballos ${ }^{2}$ (])
}

\footnotetext{
${ }^{1}$ El Colegio de la Frontera Sur. Carretera Panamericana y Periférico Sur s/n Barrio María Auxiliadora. 29290 San Cristóbal de Las Casas, Chiapas, México. * Autor para correspondencia (cavaldez@ecosur.edu.mx)

${ }^{2}$ Universidad Veracruzana, Campus para la Cultura, las Artes y el Deporte. Av. Culturas Veracruzanas 101, Col. Emiliano Zapata. 91090 Xalapa, Veracruz, México.
}

\section{RESUMEN}

El helecho Pteridium aquilinum es una de las especies invasoras más importantes del mundo. En México, en el área de influencia de la reserva de la Biosfera de Calakmul (REBICA), P. aquilinum invade y domina amplias extensiones de terrenos agrícolas y ganaderos, lo cual lleva a los campesinos a buscar nuevos sitios para cultivar, en detrimento de las áreas con selvas conservadas y protegidas. En esta investigación se evaluaron las propiedades químicas y biológicas del suelo en áreas invadidas por helechales y en vegetación madura. Para ello, se establecieron 12 parcelas con tres condiciones contrastantes, con cuatro repeticiones por condición: helechales jóvenes (HJ; 5-10 años) y viejos (HV; más de 25 años) y vegetación madura (VM). En el suelo se evaluaron los componentes químicos: $\mathrm{pH}$, materia orgánica (MO), carbono orgánico $(\mathrm{CO})$, nitrógeno total (NT) y fósforo disponible Olsen (P) $\mathrm{y}$ biológicos (acumulación de mantillo, rizomas y macrofauna). En los componentes químicos del suelo, se encontraron diferencias significativas en el $\mathrm{pH}$ $(\mathrm{F}=10.719 ; P<0.05)$ у $\mathrm{P}(\mathrm{F}=14.377 ; P<0.05)$ de HJ y HV con respecto a la VM. En los atributos biológicos se encontró que la materia seca del mantillo y de los rizomas muertos del helecho y su profundidad varió significativamente $(\mathrm{F}=5.53, P<0.05$; $\mathrm{T}=3.04, P<0.05 ; \mathrm{T}=2.57, P<0.05)$ entre HJ y $\mathrm{HV}$, respectivamente. Sin embargo, la abundancia de

Cita recomendada:

Valdez-Ramírez, C., S. I. Levy-Tacher' ${ }^{1}$, N. S. León-Martínez'1, D. A. Navarrete-Gutiérrez ${ }^{1}$ y Á. I. Ortiz Ceballos. 2020. Cambios químicos y biológicos del suelo provocados por Pteridium aquilinum (L.) Kuhn en áreas de influencia de la reserva de la biosfera de Calakmul, Campeche. Terra Latinoamericana 38: 289-300.

DOI: https://doi.org/10.28940/terra.v38i2.464 la macrofauna fue semejante entre las tres condiciones estudiadas (HV, HV y VM). De acuerdo a las variables edáficas estudiadas se encontró que la condición de HV no está degradada y que incluso presenta condiciones favorables para llevar a cabo acciones de restauración/ rehabilitación.

Palabras clave: fertilidad del suelo, invasión, macrofauna, restauración/rehabilitación, rizomas.

\section{SUMMARY}

The fern Pteridium aquilinum is one of the most important invasive species in the world. In Mexico, in the influence area of the Calakmul Biosphere Reserve (REBICA) this species invades and dominates agricultural and livestock land, which leads the peasants to look for new sites for their cultivation, to the detriment of the conserved areas. In this research, chemical and biological properties of soil were evaluated in areas invaded with bracken fern forests and in mature vegetation. Thus, 12 plots with three contrasting conditions, with four repetitions per condition were established: young helechal (HJ; 5-10 years old) and old helechal (HV, more than 25 years old) and mature vegetation $(\mathrm{VM})$. Chemical components were evaluated in the soil: $\mathrm{pH}$, organic matter (MO), organic carbon (CO), total nitrogen and available phosphorus Olsen (P), and biological component like: litter accumulation,

Recibido: 17 de noviembre de 2018 Aceptado: 06 de febrero de 2020. Publicado en Terra Latinoamericana 38: 289-300. 
rhizoms and macrofauna. In chemical components of the soil, significant differences were found in $\mathrm{pH}$ $(\mathrm{F}=10.719, P \leq 0.05)$ and $\mathrm{P}(\mathrm{F}=14.377, P \leq 0.05)$ of $\mathrm{HJ}$ and $\mathrm{HV}$ in regard to VM. In biological attributes, it was encountered that the dry matter of litter and the dead rhizomes and its depth varied significantly $(\mathrm{F}=5.53, P$ $<0.05 ; \mathrm{T}=3.04, P<0.05 ; \mathrm{T}=2.57, P<0.05)$ between $\mathrm{HJ}$ and $\mathrm{HV}$, respectively. Nevertheless, abundance of macrofauna was similar to the three conditions (HV, HJ y VM). In accordance with the edaphic variables studied, it was found that the condition $\mathrm{HV}$ is not degraded and even presents favorable conditions to carry out restoration/rehabilitation actions.

Index words: soil fertility, invasion, macrofauna, restoration / rehabilitation, rhizomes.

\section{INTRODUCCIÓN}

La invasión de helechos del género Pteridium se remonta a inicios del siglo pasado; desde entonces generó problemas en los sistemas productivos campesinos, que recientemente incrementaron por la intensificación del uso de la tierra (Minore, 1953'; Ortega, 1989; Milligan et al., 2018). La especie más representativa del género es Pteridium aquilinum L. Kuhn. En la región tropical y templada, la invasión y dominancia del helecho en grandes extensiones dificulta el aprovechamiento agropecuario y tiene como consecuencia la interrupción del proceso de la sucesión vegetal (Pakeman et al., 2000; Samecka et al., 2009; CONABIO, 2013; Berget et al., 2015), pues se sabe que la permanencia del helecho puede alcanzar hasta 200 años (Crane, 1990). Por esta razón, cuando los helechales invaden los campos de cultivo, los campesinos generalmente deciden abandonar sus terrenos debido a la dificultad que implica su erradicación (manual, mecánica y química) (Peñaloza, 2008²; Suazo, et al., 2015). P. aquilinum presenta características que le permiten un gran éxito reproductivo, por ejemplo, tiene un sistema de rizomas profundos que favorecen su persistencia (Schneider, 2004a, b3; Schneider, 2006). Logra prosperar en suelos pobres en nutrientes, bajo sombra y principalmente bajo luz solar directa en donde tiene su mayor éxito reproductivo (Marrs y Watt, 2006; Green y Nishimura, 2011; Suazo et al., 2015).

Además, gracias a la alta capacidad de almacenamiento de nutrientes en sus rizomas, rápida producción de frondas y el desarrollo de un dosel denso; esta especie se propaga y domina en una amplia gama de condiciones, inclusive en suelos ácidos (Marrs y Watt, 2006; Adame, 2011). Como todos los helechos, su reproducción es mediante esporas que por su tamaño pequeño le confiere una alta capacidad de dispersión (Schneider, 2004b ${ }^{3}$ ). Asimismo, su germinación es favorecida por la presencia del fuego (Colleen, 20114; Ramírez et al., 2010). Estas situaciones hacen favorable su distribución en todos los continentes, excepto en la Antártida (Green y Nishimura, 2011), desiertos y montañas altas (Marrs y Watt, 2006).

Otra propiedad del helecho, que le permite una mayor competencia y desarrollo, es su capacidad para producir compuestos químicos como el cianuro de hidrógeno $(\mathrm{HCN})$, que le sirve de protección hacia los patógenos o herbívoros (Crane, 1990). También sintetiza sustancias como los taninos, sesquiterpenos y flavonoides que pueden funcionar como aleloquímicos (Jones y Firn, 1979). Esta situación proporciona a P. aquilinum un efecto de inhibición sobre el crecimiento de otras plantas; no obstante, aún faltan pruebas para comprender de forma más clara este mecanismo de inhibición de la germinación de semillas y desarrollo de las plantas que crecen junto a P. aquilinum (Marrs y Watt, 2006). Cuando la colonización de los helechales es exitosa, induce un proceso de transformación de los ecosistemas del suelo que aún no es muy claro, pues los conocimientos al respecto aún son incipientes (DeLuca et al., 2013). En este sentido, existe información contrastante sobre el efecto que el helecho genera sobre el suelo para degradarlo y volverlo improductivo, o si por el contrario puede favorecer eventos que benefician la fertilidad edáfica mediante la aireación favorecida por el crecimiento de sus rizomas y su asociación

\footnotetext{
${ }^{1}$ Minore, D. 1953. The growth of Pteridium aquilinum (L.) Kuhn vari. pubescens in relation to Geographic source, soil nutrients, and forest site. Tesis. University of California, Berkeley.

${ }^{2}$ Peñaloza Guerrero, C. B. 2008. Efecto de sombra y estrés mecánico al rizoma de Pteridium aquilinum, un helecho invasor en los trópicos de México. Tesis de Maestría. Postgrado en Ciencias Biológicas. Centro de Investigaciones en Ecosistemas. Universidad Nacional Autónoma de México. 51 p.

${ }^{3}$ Schneider, L. 2004b. Understanding bracken fern (Pteridium aquilinum (L.) Kuhn) invasion in the Southern Yucatán Peninsular Region through Integrated Land-Change Science [Graduate School of Geography]. Ph. D. diss., Faculty of Clark University. Worcester, MA, USA.

${ }^{4}$ Colleen, E. 2011. Characterizing invasive species: the case of bracken fern (Pteridium aquilinum) in the Mesoamerican Biological Corridor Sian Ka'anCalakmul, México. A thesis submitted to the Graduate School- New Brunswick Rutgers, The State University of New Jersey in partial fulfillment of the requirements for the degree of Master of Science Graduate Program in Geography. New Brunswick, NJ, USA.
} 
con micorrizas. Por un lado se ha documentado la influencia de $P$. aquilinum en procesos favorables para el suelo; por ejemplo: la reducción de la podzolización y acidez (Marrs y Watt, 2006; DeLuca et al., 2013), así como el incremento en la nitrificación (DeLuca et al., 2013). En contraparte, hay investigaciones en las que la colonización del helecho se relaciona con la degradación del suelo y del sitio debido a un complejo proceso social y productivo (Schneider, 2006). En terrenos colonizados por helechales disminuye notablemente la riqueza de especies vegetales del sotobosque, lo que resulta en un valor de conservación menor con relación al estado previo a la invasión (Milligan et al., 2018). No obstante, es conveniente tomar en cuenta las formas de manejo que favorecen la invasión del helecho, como los regímenes del uso del fuego que promueven la persistencia y dominancia de $P$. aquilinum en áreas agropecuarias (Schneider, 2006).

Cuando se habla de conservación ecológica, la degradación del suelo es uno de los principales desafíos para la sostenibilidad de los sistemas agropecuarios (Doi y Sakurai, 2004). En todo el mundo se están realizando trabajos orientados a recuperar las condiciones productivas en los sitios que sufrieron procesos de degradación a causa de la llegada de Pteridium aquilinum, y que actualmente están en desuso (Pakeman et al., 2002; Ghorbani et al., 2003; Levy et al., 2015; Milligan et al., 2018).

Una de las causas de la degradación productiva del suelo es la pérdida de su macrofauna, debido a que la presencia de estos organismos se considera un buen indicador de la calidad ecológica de un sitio (Cabrera, 2012). A este grupo biótico lo constituyen organismos mayores a $2 \mathrm{~mm}$ que participan en los procesos de fragmentación de partículas fecales, la estimulación de la producción microbiana, además de influir en el ciclo de la materia orgánica y de los nutrientes del suelo (Zerbino, 20055; Cabrera, 2012). En este sentido, la edad del helechal puede ser un factor importante para detectar patrones de degradación del suelo.

Cuando ocurren cambios en la estructura y composición física y química del suelo, existe un impacto negativo sobre la macrofauna presente en el sitio debido a la alta sensibilidad de estos organismos a dichos cambios (Cabrera, 2012). En este sentido, $P$. aquilinum contribuye en la transformación de los ecosistemas terrestres, pero la información al respecto es incipiente (DeLuca et al., 2013), y no se sabe a ciencia cierta el impacto que podría tener la presencia prolongada del helecho en algunos cambios químicos y de la macrofauna del suelo en los sitios invadidos. Una de las principales razones de la acumulación de la gruesa capa de frondas en los helechales viejos, responde a la baja cantidad de detritívoros del suelo. De esta manera, es posible encontrar frondas que durante décadas persisten sin degradarse (Marrs y Watt, 2006). Al respecto, se ha evaluado el grosor del colchón de frondas muertas acumulado bajo el dosel del helecho (Ghorbani et al., 2006) y se han hecho estimaciones de la materia seca generada por las frondas del helecho por año (Rasmussen et al., 2003), pero no se ha precisado el peso de la materia seca bajo distintas etapas de desarrollo del helecho (joven y viejo).

En el marco de las aseveraciones anteriores, se realizó esta investigación en la comunidad Ing. Ricardo Payró Jene, del municipio de Calakmul, con el propósito de evaluar las características químicas y biológicas del suelo en helechales y vegetación madura que coadyuven a comprender los posibles efectos de esta especie en los procesos de degradación de las áreas agropecuarias. Con este propósito, se estudiaron los componentes químicos ( $\mathrm{pH}, \mathrm{MO}, \mathrm{CO}, \mathrm{NT}$ y $\mathrm{P})$ y biológicos (mantillo, materia seca de rizomas vivos y muertos; y macrofauna: detritívoros e ingenieros del suelo) en 12 parcelas partiendo de la historia de uso de cada sitio invadido por $P$. aquilinum. Se consideró además, un gradiente temporal de degradación por causa del helecho: helechal viejo y helechal joven; así como vegetación madura como referente. Con ello surge la siguiente pregunta: ¿Existe degradación química y biológica del suelo a consecuencia del establecimiento prolongado de Pteridium aquilinum en parcelas agrícolas en desuso?

\section{MATERIALES Y MÉTODOS}

El ejido Ing. Ricardo Payró Jene, pertenece al municipio de Calakmul, Campeche (Figura 1) y es parte de la zona de influencia de la Reserva de la Biosfera de Calakmul (19० $15^{\prime}$ y $17^{\circ} 45^{\prime} \mathrm{N}$ y $90^{\circ} 10^{\prime}$ y $89^{\circ} 15^{\prime}$ O). A su vez, la región de Calakmul forma parte de la Península de Yucatán (PY) que se distingue ${ }_{5}^{5}$ Zerbino-Bardier, M. S. 2005. Evaluación de la densidad, biomasa y diversidad de la macrofauna del suelo en diferentes sistemas de producción. Tesis de
Maestría. Universidad de la República. Facultad de Ciencias. Uruguay. 
por presentar una geología de tipo kárstico con tierras que van desde los $100 \mathrm{~m}$ hasta $\operatorname{los} 350 \mathrm{~m}$ de altitud. En la región de Calakmul, los suelos predominantes son principalmente rendzinas (molisoles) (Schneider, $\left.2004 b^{3}\right)$. La temperatura se presenta con rango de 24 a $28{ }^{\circ} \mathrm{C}$ (INEGI, 2009). La vegetación predominante para la zona de estudio es selva mediana subcaducifolia (Miranda y Hernández-X., 1963) con períodos de inundación a lo largo del año (SEMARNAP, 1999). La región tiene un clima caliente subhúmedo con lluvias en verano "Aw", de acuerdo a la clasificación de Köeppen modificada por Enriqueta García (García, 2004).

Ing. Ricardo Payró Jene es un poblado rural que cuenta con extensión total de 5335 ha y 96 ejidatarios. Las principales actividades productivas son la milpa y la apicultura, más recientemente el cultivo mecanizado de chile jalapeño y maíz en pequeñas superficies (20 ha). Los habitantes son procedentes de por lo menos seis estados del país y se distribuyen en una zona urbana habitacional de 37.5 ha. La selección de esta localidad para realizar la investigación fue debido a las grandes extensiones de áreas invadidas por los helechales
(350 ha), así como por la disposición de los campesinos locales para colaborar en esta investigación.

Previo al establecimiento de las parcelas de muestreo, en los sitios invadidos por el helecho, con cada uno de los propietarios se indagó sobre el historial de uso a partir de la vegetación madura y hasta la llegada de $P$. aquilinum (Suazo et al., 2015). Con esta información fue posible reconocer la presencia histórica del helecho en cada una de las áreas, a partir de lo cual se eligieron 12 parcelas con una superficie de $400 \mathrm{~m}^{2}$ $(20 \times 20 \mathrm{~m})$ cada una, considerando tres condiciones con cuatro repeticiones por condición: a) vegetación madura "VM", b) helechales viejos "HV" (más de 25 años), y c) helechales jóvenes "HJ" (5-10 años) (Figura 1). También se consideró que las parcelas no estuvieran sometidas a mecanización (uso de tractor) y que el terreno fuera planada (rendzinas), evitando la presencia de altillos pedregosos, clasificados como suelos de tipo vertisol y leptosol, respectivamente (FAO, 2015). Por último, cada repetición por condición fue de un propietario distinto con la finalidad de cumplir los supuestos de independencia entre las repeticiones.

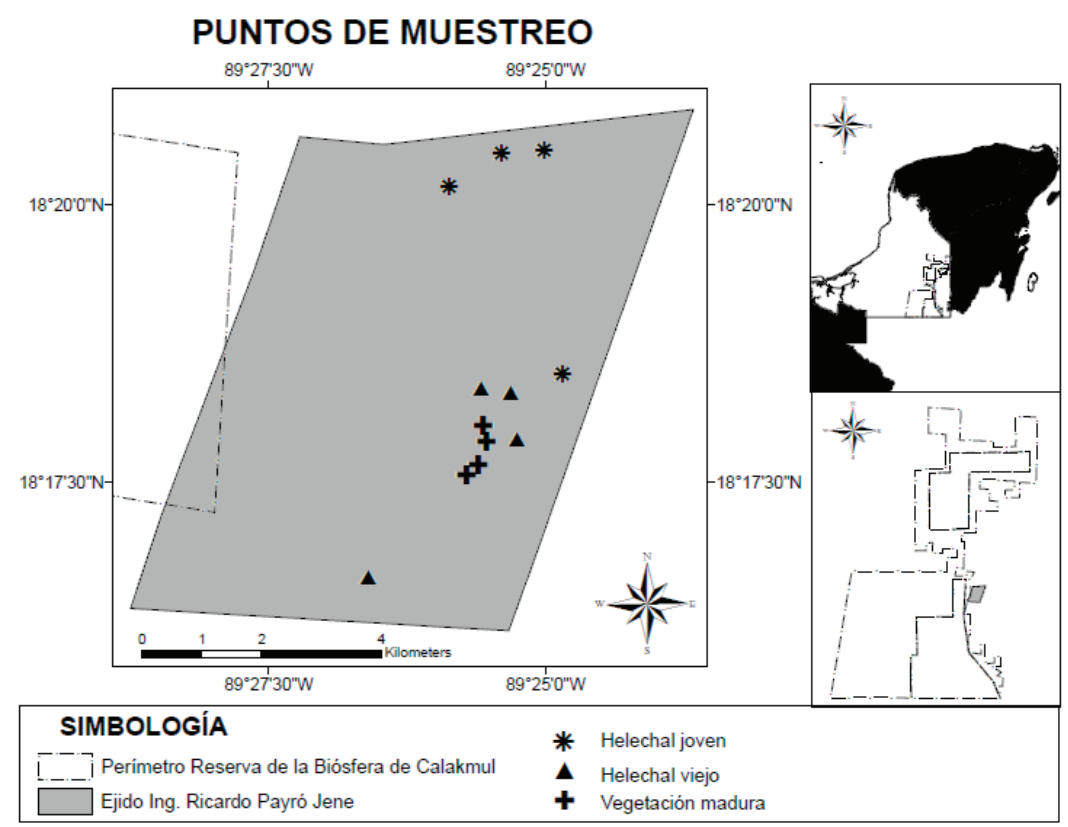

Figura 1. Localización de las parcelas para la evaluación química y biológica del suelo en el ejido Ing. Ricardo Payró Jene, zona de influencia de la reserva de la biosfera de Calakmul.

Figure 1. Location of the plots for chemical and biological evaluation of the soil in the Ing. Ricardo Payró Jene ejido, area of influence of the Calakmul biosphere reserve. 
La textura de los suelos en las tres condiciones estudiadas fue de arcillosa a franco limosa con una altitud de las parcelas que osciló entre 230 - $280 \mathrm{~m}$.

\section{Variables Químicas del Suelo}

En cada una de las parcelas se ubicaron cinco puntos distribuidos de acuerdo al método "cinco de oro". En cada punto se tomaron dos muestras con un nucleador de $2.54 \mathrm{~cm}$ de diámetro que se introdujo de manera vertical a profundidad de $25 \mathrm{~cm}$ con previa remoción del humus (horizonte 0). Las 10 muestras fueron homogeneizadas en una bolsa de nylon para obtener una muestra compuesta de $800 \mathrm{~g}$. En el "Laboratorio de Suelos y Plantas" de ECOSUR, Unidad San Cristóbal de Las Casas, las muestras fueron preparadas. Para ello, fueron secadas al aire libre durante $48 \mathrm{~h}$, se retiraron las piedras y material orgánico visible y se continuó con la molienda y tamizado. Para el análisis de $\mathrm{pH}$ y fósforo disponible (método Olsen) se usó malla 10 (2 mm), y malla $40(0.5 \mathrm{~mm})$ para las variables materia orgánica, carbono orgánico y nitrógeno total. Para el análisis del suelo, las muestras fueron entregadas al personal del laboratorio. Las técnicas para procesar y analizar el suelo fueron: método AS-02 para $\mathrm{pH}$, combustión húmeda ácido/dicromato para el carbono orgánico, método AS-07 de Walkley y Black para materia orgánica, técnica micro-Kjeldahl para nitrógeno total y método Olsen (utilizando $\mathrm{NaHCO} 30.5 \mathrm{M}$, $\mathrm{pH} 8,5)$ para fósforo disponible; todo con base en la Norma Oficial Mexicana NOM-021-RECNAT-2000 (SEMARNAT, 2002).

\section{Evaluación del Mantillo}

En cada una de las 12 parcelas se obtuvo una muestra de la capa de hojarasca acumulada o mantillo. Con base en Anderson e Ingram (1993) se delimitó un cuadrante de $25 \times 25 \mathrm{~cm}$ dentro del cual se colectó el material hasta llegar al suelo. Las muestras fueron secadas durante $48 \mathrm{~h}$ a temperatura promedio de $55^{\circ} \mathrm{C}$ en el cuarto de hornos y estufas de ECOSUR-Unidad San Cristóbal. Por último, se pesaron en seco con una escala de precisión de $0.1 \mathrm{~g}$.

\section{Materia Seca y Profundidad de Rizomas}

En cinco áreas de $25 \times 25 \mathrm{~cm}$ asignadas al azar, dentro de cada una de las 12 parcelas, se excavó un orificio tan profundo como el rizoma más profundo $(\sim 0.24 \mathrm{~m})$ y se cuantificaron los rizomas de helecho vivos y muertos. Las muestras colectadas de rizomas se rociaron con agua para eliminar restos de lodo (Levy et al., 2015) y se secaron en estufa a $55^{\circ} \mathrm{C}$ para después ser pesadas con escala de precisión digital.

\section{Macrofauna del Suelo}

Los muestreos de la macrofauna del suelo fueron realizados en el mes de julio, después de que iniciaron las lluvias (Colleen, 2011) ${ }^{4}$, época en que los organismos del suelo presentan mayor actividad (Huerta et al., 2008). Con el método de muestreo del Tropical Soil Biology and Fertility Programme (TSBF) se realizó la colecta de los macroinvertebrados (Gálvez et al., 2016). En cada una de las parcelas se tomó un monolito de $25 \times 25 \times 30 \mathrm{~cm}$, de los cuales se obtuvieron todos los organismos para ser fijados con alcohol al 70\%. Los muestreos se tomaron a una distancia de $90 \mathrm{~cm}-120 \mathrm{~cm}$ del punto central de cada parcela. La separación en grandes grupos taxonómicos y gremios se realizó con base en algunos trabajos previos (Brussaard, 1998; Morón y Huerta 2006; Huerta et al., 2008).

\section{Análisis Estadísticos}

Todos los análisis se realizaron utilizando el software SPSS versión 21 (Corporation, 2012). Se realizó la extrapolación de datos: biomasa de hojarasca y materia seca de rizomas $\left(\mathrm{kg} \mathrm{m}^{-2}\right)$; macrofauna (número de organismos $\mathrm{m}^{-2}$ ).

Para el análisis estadístico, de la información recabada, se corroboró la normalidad a partir de la prueba Shapiro - Wilks y homocedasticidad de los datos. Para los datos de los factores de respuesta: componentes químicos, mantillo y materia seca, y profundidad de rizomas; se aplicaron pruebas de $\mathrm{t} \mathrm{o}$ análisis de varianza (ANOVA), con prueba post hoc de Tukey, en donde las condiciones de muestreo fueron las variables independientes. Los datos de macroinvertebrados se transformaron a logaritmos de base $10\left(\log _{10}(\mathrm{x}+1)\right)$, para posteriormente analizarlos con ANOVA. En este caso los factores de respuesta fueron las abundancias para cada uno de los gremios colectados y las condiciones de muestreo fueron las variables independientes. 


\section{RESULTADOS Y DISCUSIÓN}

\section{Variables Químicas del Suelo}

De las variables del suelo estudiadas en las tres condiciones consideradas (vegetación madura, helechales viejos y helechales jóvenes), el fósforo disponible $\left(\mathrm{mg} \mathrm{kg}^{-1}\right)$ y $\mathrm{pH}$ (escala logarítmica 0 -14) fueron las únicas que mostraron diferencias significativas $(\mathrm{F}=14.38 ; P \leq 0.05$ y $\mathrm{F}=10.72$; $P \leq 0.05$ respectivamente) (Cuadro 1). El contenido de carbono orgánico, materia orgánica y nitrógeno total fue semejante entre las condiciones; además, cabe notar que el HV tuvo los valores medios más altos para todas las variables, excepto para $\mathrm{pH}$ que fue mayor para HJ (Cuadro 1).

El aprovechamiento de la selva a partir de su uso agrícola, conlleva a un decremento de nutrientes del suelo como el nitrógeno total (NT) y fósforo disponible (P) (Huerta et al., 2008). Los resultados mostraron que el mantillo, MO, CO, NT y P del suelo en el HV, presentaron valores superiores a los alcanzados en HJ y VM. Quizá lo anterior se debe a que en vegetación secundaria existen mecanismos para una rápida adquisición de nutrientes por las plantas; en este caso, la especie que aprovecha los nutrientes es $P$. aquilinum. Posteriormente, en estadios tardíos, la estrategia de aprovechamiento de los nutrientes por las plantas, es inversa; es decir, estos se conservan en la vegetación exuberante y disminuyen en el suelo (Hernández, 2019). Sin embargo, aunque en HV existe mayor fertilidad en el suelo por la alta acumulación de frondas, es ésta misma condición la que no permite que otras especies vegetales puedan usar los nutrientes para crecer y competir en el sitio. Cabe destacar la importancia del HV para restituir la fertilidad del suelo y la restauración de su biota, incluso a niveles superiores alcanzados por la condición de referencia, es decir la VM. La alta fertilidad en los suelos generada por la presencia prolongada de los helechales, ha sido documentada en el Reino Unido donde un área dominada por el helecho fue tratada durante dos décadas, dando lugar decremento de los niveles de MO, C, NT y P del suelo a consecuencia del establecimiento de la vegetación nativa (Milligan et al., 2018). Por su parte, la mayor concentración de NT y P en HV respecto a HJ y VM, quizá se deba a que el $\mathrm{HV}$ fue la condición con la mayor presencia de lombrices de tierra (Clitellata) (Para ver detalles consultar apartado "composición de macroinvertebrados del suelo"). Estos organismos favorecen a que exista más nitrógeno disponible en forma de amonio $\left(\mathrm{NH}_{4}\right)$ en el suelo (He et al., 2018). De esta manera, los resultados obtenidos sugieren que la habilidad competitiva del helecho puede estar asociada a la presencia de las lombrices, pero también a que HV fue la condición con mayor disponibilidad de mantillo por la constante acumulación de frondas del helecho. Por otro lado, Huerta et al. (2008) encontraron una relación similar entre la mayor presencia de lombrices de tierra y los niveles más altos para NT y $\mathrm{P}$, pero en este caso no en helechales, sino en vegetación madura y policultivos.

Los valores de MO del suelo pueden ser un parámetro útil para evaluar la degradación de un sitio

Cuadro 1. Evaluación de los principales componentes químicos del suelo en helechales jóvenes (HJ), viejos (HV) y vegetación madura (VM), ubicados en el ejido Ing. Ricardo Payró Jene.

Table 1. Evaluation of the main chemical components of the soil in young ferns (HJ), old (HV) and mature vegetation (VM), located in the ejido Ing. Ricardo Payró Jene.

\begin{tabular}{|c|c|c|c|c|c|}
\hline \multirow{2}{*}{ Variable } & \multicolumn{5}{|c|}{ Variables químicas del suelo } \\
\hline & $\mathrm{pH}$ & $\mathrm{MO}$ & $\mathrm{CO}$ & NT & $\mathrm{P}$ \\
\hline & $0-14$ & $\ldots \ldots$ & $\cdots \mathrm{g} \mathrm{kg}^{-2} \ldots$ & $\cdots \cdots$ & $\mathrm{mg} \mathrm{kg}{ }^{-2}$ \\
\hline VM & $7.08 \pm 0.43 \mathrm{a}$ & $95.08 \pm 27.51 \mathrm{a}$ & $55.15 \pm 15.96 \mathrm{a}$ & $3.30 \pm 0.98 \mathrm{a}$ & $2.62 \pm 1.43 \mathrm{a}$ \\
\hline $\mathrm{HV}$ & $7.61 \pm 0.18 \mathrm{ab}^{\dagger}$ & $115.23 \pm 25.23 \mathrm{a}$ & $66.84 \pm 14.63 \mathrm{a}$ & $5.12 \pm 1.82 \mathrm{a}$ & $6.78 \pm 1.11 \mathrm{~b}$ \\
\hline HJ & $7.96 \pm 0.07 b$ & $90.56 \pm 26.03 \mathrm{a}$ & $52.53 \pm 15.10 \mathrm{a}$ & $3.47 \pm 1.56 \mathrm{a}$ & $4.20 \pm 0.64 \mathrm{ab}$ \\
\hline
\end{tabular}

En el pH se usa la escala logarítmica de 0-14; $\mathrm{MO}=$ materia orgánica; $\mathrm{CO}=$ carbono orgánico; $\mathrm{NT}$ = nitrógeno total; $\mathrm{P}=$ fósforo disponible; $\mathrm{VM}$ vegetación madura; $\mathrm{HV}=$ helechales viejos; $\mathrm{HJ}=$ jóvenes. ${ }^{\dagger}$ Media $( \pm \mathrm{SD})$. Las letras diferentes entre las columnas denotan diferencias significativas (Tukey, $\left.P \leq 0.05\right)$. The $\mathrm{pH}$ uses the logarithmic scale of $0-14 ; \mathrm{MO}=$ organic matter; $\mathrm{CO}=$ organic carbon; $\mathrm{NT}=$ total nitrogen; $\mathrm{P}=$ available phosphorus; $\mathrm{VM}=$ mature vegetation; $\mathrm{HV}=$ old ferns; $\mathrm{HJ}=$ young. ${ }^{\dagger}$ Average $( \pm \mathrm{SD})$. The different letters between the columns denote significant differences (Tukey, $\left.P \leq 0.05\right)$. 
(Velasquez et al., 2007). De hecho, la productividad de los suelos tropicales en gran medida se debe a la materia orgánica acumulada por los ecosistemas originales (Huerta et al., 2008), así como por el uso de buenas prácticas agropecuarias sustentables (Kloster et al., 2016). En los presentes resultados se reconoció una tendencia a que la cantidad de la $\mathrm{MO}$ fuera mayor para HV $\left(115.23 \pm 25.23 \mathrm{~g} \mathrm{~kg}^{-1}\right)$ con relación a VM $\left(95.08 \pm 27.51 \mathrm{~g} \mathrm{~kg}^{-1}\right)$ y $\mathrm{HJ}\left(90.56 \pm 26.03 \mathrm{~g} \mathrm{~kg}^{-1}\right)$. Estos hallazgos para MO en $\mathrm{HV}$ y $\mathrm{HJ}$, coinciden con los valores de acumulación referidos por Marrs y Watt (2006). Ellos mencionan que en sitios colonizados por el helecho, la cantidad de MO puede presentar una amplia oscilación, entre 10 - $920 \mathrm{~g} \mathrm{~kg}^{-1}$ de suelo colectado, dependiendo de la variación de las condiciones ambientales que favorezcan o no al helecho.

En cuanto al $\mathrm{pH}$, la mayor alcalinidad de los suelos en los helechales (HJ: $7.96 \pm 0.07$; HV: $7.61 \pm 0.18$ ) respecto a la VM (7.08 \pm 0.43$)$, quizá responda a la acumulación de mantillo a partir de las frondas. Marrs y Watt (2006) reportaron que, en el proceso de descomposición de las frondas en el suelo, viene acompañado de la liberación de abundantes cantidades de cationes, los cuales se lixivian rápidamente y reducen la acidez de la tierra. La menor alcalinidad de HV respecto a HJ quizá se deba a la mayor acumulación de mantillo y de MO en HV. Previamente se reportó que algunos componentes de la descomposición de las frondas del helecho (grupos carboxílicos y fenólicos), funcionan como ácidos débiles y pueden disminuir el pH del suelo, aunado a que en suelos casi neutros o formados por gran cantidad de carbonatos, el $\mathrm{pH}$ disminuye por causa de la presión del $\mathrm{CO}_{2}$ en el suelo (Martínez et al., 2008).

\section{Materia Seca y Profundidad de Rizomas}

La materia seca de los rizomas muertos mostró diferencias significativas entre $\mathrm{HV}$ y $\mathrm{HJ}$ con valores $\mathrm{t}=3.04 ; P \leq 0.05$ (Cuadro 2). La condición HV tuvo $145 \%$ más cantidad de rizomas muertos comparado con HJ. Por su parte, para la materia seca de los rizomas vivos, no se encontraron diferencias significativas, pero sí numéricas con base en los valores medios. HJ presentó $36 \%$ más rizomas $\left(16 \mathrm{Mg} \mathrm{ha}^{-1}\right)$ con respecto a HV (Cuadro 2). Para la profundidad de los rizomas, se identificaron diferencias significativas entre $\mathrm{HV}$ y $\mathrm{HJ}$ con valores $\mathrm{t}=2.57 ; P \leq 0.05$ (Cuadro 2 ).
Una de las características que le confiere al helecho su gran capacidad competitiva sobre otras especies, es que cuenta con un sistema de rizomas que le permite sobrevivir a la presencia del fuego y rebrotar continuamente durante un largo periodo (Schneider, 2004b ${ }^{3}$ ). Los resultados mostraron que dicha capacidad competitiva es menor para HV debido a que esta condición presenta la mayor cantidad de rizomas muertos y menos rizomas vivos respecto a $\mathrm{HJ}$ (Cuadro 2). Es probable que la mayor cantidad de rizomas muertos sea parte del proceso natural de envejecimiento del helechal. En contraste a los hallazgos del presente estudio, en bosques templados, se ha descrito un proceso diferente, en donde a consecuencia de la muerte de los rizomas y la descomposición de las frondas, se genera una rica capa de humus en la superficie del suelo, que favorece el crecimiento de los rizomas. Es decir, a diferencia de lo encontrado, en los HV de los bosques estudiados, los rizomas migran hacia la capa superficial (humus) y se vuelven vulnerables a las heladas y sequías que disminuyen su producción de frondas; este proceso puede tardar desde unas cuantas décadas (Marrs y Watt, 2006) o hasta 200 años (Crane, 1990) y una vez que acontece, comienza la colonización de leñosas, que ya no son suprimidas por la presencia del helecho y la sucesión vegetal continúa (Marrs y Watt, 2006).

Cuadro 2. Producción de materia seca y profundidad de rizomas (vivos y muertos) en helechales jóvenes (HJ) y viejos (HV) ubicados en el ejido Ing. Ricardo Payró Jene.

Table 2. Production of dry matter and depth of rhizomes (live and dead) in young (HJ) and old (HV) ferns located in the Ing. Ricardo Payró Jene ejido.

\begin{tabular}{lccc}
\hline \multirow{2}{*}{ Variable } & \multicolumn{3}{c}{ Materia seca y profundidad de rizomas } \\
\cline { 2 - 4 } & \multicolumn{2}{c}{ MSeRiVi } & \multicolumn{2}{c}{ MSeRiMu } & Prof \\
\hline & $-\ldots-\ldots \mathrm{kg} \mathrm{m}^{-2}-\ldots \ldots$ & $\mathrm{cm}$ \\
HV & $1.012 \pm 0.275 \mathrm{a}^{\dagger}$ & $0.648 \pm 0.189 \mathrm{~b}$ & $23.20 \pm 1.23 \mathrm{~b}$ \\
HJ & $1.587 \pm 0.503 \mathrm{a}$ & $0.264 \pm 0.165 \mathrm{a}$ & $19.25 \pm 2.81 \mathrm{a}$ \\
\hline
\end{tabular}

$\overline{M S e R i V i}=$ materia seca de rizomas vivos; MSeRiVi = materia seca de rizomas muertos; Prof = profundidad; $\mathrm{HV}=$ helechales viejos; $\mathrm{HJ}=$ jóvenes.

${ }^{\dagger}$ Media $( \pm \mathrm{SD})$. Las letras diferentes entre las columnas denotan diferencias significativas (t-student, $P \leq 0.05$ ).

MSeRiVi $=$ dry matter of live rhizomes; MSeRiVi $=$ dry matter of dead rhizomes; Prof $=$ depth; $\mathrm{HV}=$ old ferns; $\mathrm{HJ}=$ youth. ${ }^{\dagger}$ Average $( \pm \mathrm{SD})$. The different letters between the columns denote significant differences (t-student, $P \leq 0.05$ ). 


\section{Hojarasca o Mantillo}

Existe mayor cantidad de mantillo en el HV con respecto al $\mathrm{HJ}(\mathrm{F}=5.53 ; P \leq 0.05)$. El valor medio más alto fue para $\mathrm{HV}$ con $2.87 \pm 1.05 \mathrm{~kg} \mathrm{~m}^{-2}$ de hojarasca y el más bajo para HJ con $1.52 \pm 0.07 \mathrm{~kg} \mathrm{~m}^{-2}$. La VM $\mathrm{y}$ el $\mathrm{HV}$ no presentaron diferencias significativas, con valor de biomasa de hojarasca promedio de $2.24 \pm 0.19 \mathrm{~kg} \mathrm{~m}^{-2}$.

De manera específica para HVy HJ, su acumulación de mantillo en el suelo, coincide con lo descrito por DeLuca et al. (2013). Ellos reportaron que la producción de mantillo de las frondas del helecho puede resultar en acumulación de hojarasca de 1 y $5 \mathrm{~kg} \mathrm{~m}^{-2}$. A su vez, la mayor acumulación de mantillo en $\mathrm{HV}(2.87 \pm 1.05)$ respecto a $\mathrm{HJ}(1.52 \pm 0.07)$, quizá se deba a que las frondas tienen una tasa de descomposición muy lenta, lo que causa su mayor acumulación en el suelo. Al respecto, se ha descrito que la tasa de acumulación de frondas del helecho en el suelo, es mayor que la tasa de descomposición de las mismas, que puede durar hasta 11 años, dependiendo de las condiciones ambientales y de la presencia de la macrofauna del suelo (Marrs y Watt, 2006).

\section{Composición de Macroinvertebrados del Suelo}

Se muestreó un total de $4800 \mathrm{~m}^{2}$ de suelo en donde se encontraron 619 macroinvertebrados que correspondieron a 9 clases, 15 órdenes y 10 familias (Cuadro 3). De estos, 12 individuos (1.9\%) fueron determinados hasta género, $95(15.3 \%)$ a nivel de familia, $421(68.0 \%)$ hasta orden y $619(100 \%)$ a nivel de clase. En VM, las clases dominantes fueron Gastropoda, Insecta y Clitellata, que representaron $74 \%$ del total de especímenes. Para el caso de HV, las clases más representativas fueron Clitellata e Insecta con $72 \%$ del total de colectas; por último, para HJ fueron Gastropoda e Insecta con $87 \%$ del total de organismos.

Desde la perspectiva de los gremios, $44.1 \%$ correspondieron a detritívoros (273 individuos), 37.6\% a ingenieros del suelo (233), 12.6\% a depredadores (78), $0.5 \%$ a parásitos (3), $0.3 \%$ a herbívoros (2) y $4.8 \%$ a otros (30). Los detritívoros e ingenieros del suelo fueron los más representativos y en conjunto corresponden $81.7 \%$ del total de ejemplares (Figura 2).
Análisis de Medias para la Abundancia Total de Macroinvertebrados del Suelo Colectados en VM, HV y HJ

El análisis de varianza de un factor no mostró diferencias significativas para la abundancia de la macrofauna edáfica colectada entre la vegetación madura, helechales viejos y jóvenes $(P>0.05)$. El valor medio más alto fue para $\mathrm{HJ}$, seguido de $\mathrm{HV}$ y $\mathrm{VM}$ con valores respectivos de $1.69,1.55$ y 1.55 . La mayor cantidad de organismos colectados en HV, quizá se deba a que es también la condición que presentó la mayor disponibilidad de recurso (materia orgánica) para macrofauna del suelo. Huerta et al. (2008) también encontraron una mayor densidad de macroinvertebrados del suelo en sitios más ricos en MO del suelo en vegetación madura y policultivos.

Es difícil comparar los grupos taxonómicos encontrados en los helechales, pues existe poca investigación sobre el tema. Para el caso de los grupos Annelida, Coleoptera, Collembola, Aranaeae, Isopoda y Pseudoscorpionida encontrados en HV y HJ, coincide con lo reportado en 1970 en áreas invadidas por helechales dentro de Lakenheath Warren, Suffolk (zona protegida de la Unión Europea), en donde se observó la presencia de estos macroinvertebrados en el mantillo del helecho (Marrs y Watt, 2006). En este estudio se confirma que dichos grupos taxonómicos se alimentan de Pteridium aquilinum.

\section{Análisis de Medias de los Detritívoros e Ingenieros del Suelo en VM, HV y HJ}

El análisis de varianza de un factor no mostró diferencias significativas para los dos gremios más abundantes: detritívoros e ingenieros del suelo entre la vegetación madura y helechales viejos y jóvenes $(P>0.05)$ (Cuadro 4).

En conjunto, los dos gremios conformaron $81.7 \%$ del total de organismos colectados. En el caso de los detritívoros, en otras investigaciones ya han reportado a este grupo con las mayores abundancias de organismos del suelo (Morón y Huerta, 2006). En general la presencia de estos organismos terrestres puede ser un indicador de buena calidad del suelo (Cabrera, 2012), que coincide con los resultados químicos obtenidos del suelo en esta investigación. 
Cuadro 3. Diversidad de macroinvertebrados del suelo en helechales jóvenes (HJ), viejos (HV) y vegetación madura (VM), ubicados en el ejido Ing. Ricardo Payró Jene.

Table 3. Diversity of soil macroinvertebrates in young ferns (HJ), old (HV) and mature vegetation (VM), located in the ejido Ing. Ricardo Payró Jene.

\begin{tabular}{|c|c|c|c|c|c|c|}
\hline Clase & Orden & Familia & $\mathrm{VM}$ & $\mathrm{HV}$ & HJ & Total \\
\hline & & & $-\cdots$ & $\ldots$ & $-\ldots$ & $\ldots$ \\
\hline Arachnida & $\begin{array}{l}\text { Pseudoscorpiones } \\
\text { Araneae }\end{array}$ & Neobisiidae & 10 & 11 & 2 & 23 \\
\hline Chilopoda & Scolopendromorpha & Scolopendridae & 13 & 2 & 7 & 22 \\
\hline Clitellata & & & 40 & 112 & 1 & 153 \\
\hline Diplopoda & Polydesmida & Polydesmidae & 0 & 2 & 8 & 10 \\
\hline Entognatha & $\begin{array}{l}\text { Diplura } \\
\text { Collembola }\end{array}$ & & 19 & 12 & 4 & 35 \\
\hline Gastropoda & Pulmonata & & 59 & 31 & 142 & 232 \\
\hline \multirow[t]{7}{*}{ Insecta } & Coleoptera & $\begin{array}{l}\text { Staphylinidae } \\
\text { Scarabeidae } \\
\text { Tenebrionidae }\end{array}$ & & & & \\
\hline & Hymenoptera & Formicidae & & & & \\
\hline & Orthoptera & Gryllidae & 49 & 47 & 37 & 133 \\
\hline & Lepidoptera & Noctuidae & & & & \\
\hline & Hemiptera & Cicadidae & & & & \\
\hline & Blattodea & & & & & \\
\hline & Dermaptera & & & & & \\
\hline Malacostraca & Isopoda & & 0 & 5 & 5 & 10 \\
\hline Symphyla & & & 1 & 0 & 0 & 1 \\
\hline Total & & & 191 & 222 & 206 & 619 \\
\hline
\end{tabular}

En lo referente al gremio de los detritívoros, estuvo constituido principalmente por la clase Clitellata (Cuadro 4) representada en su mayoría por lombrices de tierra (Oligochaeta). Estos macroinvertebrados pueden ser muy sensibles a cambios en la estructura física o química del suelo, así como por clima, alimentación y humedad; persisten en suelos húmedos y con mayor materia orgánica disponible (Cabrera, 2012). Los resultados de esta investigación indican que tanto HV y HJ son similares a la VM y que los helechales mantienen condiciones favorables para la permanencia de los detritívoros. Quizá esta situación puede ocurrir debido a la densa cobertura de frondas que los helechales ofrecen y pueden proteger al suelo de los rayos directos del sol, además de proveer alimento para los organismos edáficos como milpiés y lombrices de tierra que presumiblemente están involucrados en la descomposición del helecho (Marrs y Watt, 2006).
De acuerdo con lo encontrado hasta el momento, no se cuenta con evidencias para suponer que en los helechales (HV y $\mathrm{HJ}$ ) ocurre una degradación del suelo, de sus nutrientes o de su macrofauna. Más aún, llama la atención encontrar que los HV tienen mejor fertilidad que HJ y VM. De hecho, si se considera la clasificación que utiliza la Norma Oficial Mexicana NOM-021-RECNAT 2000 (SEMARNAT, 2002) para evaluar los componentes químicos del suelo, en general las condiciones de $\mathrm{HV}$ y HJ, están dentro de los parámetros establecidos como "buena fertilidad del suelo". En el caso de la macrofauna del suelo, la norma no la considera como indicador de la calidad de un sistema productivo. No obstante, podría ser muy conveniente que el estudio de los macroinvertebrados del suelo se incorpore en las evaluaciones de fertilidad del suelo debido a las implicaciones que tienen en los procesos ecológicos del suelo. 


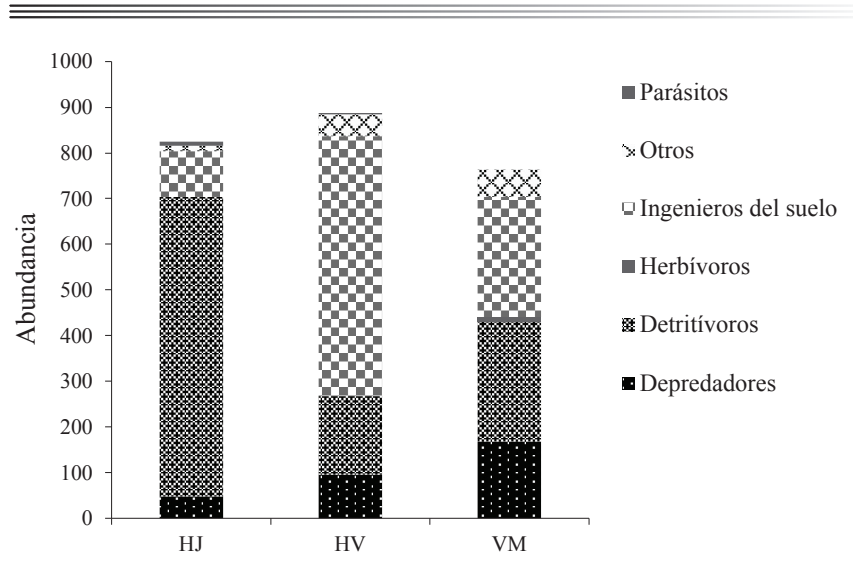

Figura 2. Abundancias de los gremios de la macrofauna del suelo por condición $\left(1600 \mathrm{~m}^{2}\right)$ : helechales jóvenes (HJ), viejos (HV) y vegetación madura (VM), ubicadas en el ejido Ricardo Payró Jene.

Figure 2. Abundances of the guilds of the soil macro-fauna by condition $\left(1600 \mathrm{~m}^{2}\right)$ : young fern $(\mathrm{HJ})$, old $(\mathrm{HV})$ and mature vegetation (VM) ferns, located in the Ricardo Payró Jene ejido.

Esta investigación se enfocó a trabajar en una de las dos condiciones edáficas existentes en la PY, las "planadas" o kankab, las cuales son codiciadas para la producción agrícola y en muchos casos, cuando son mayores a $1 \mathrm{ha}$, se utiliza maquinaria agrícola para el destronque y con esto favorecer la producción comercial. Esta es la razón principal por la cual se eligieron las planadas, por su importancia económica para la agricultura. Por otro lado, están los "altillos" o hollum, los cuales predominan dentro del paisaje yucateco y, sin embargo, no fueron considerados en esta investigación. Para tener una mejor comprensión de la importancia de los helechales en la PY, es conveniente realizar estudios similares al presente dentro de los altillos, así como en las planadas que han sido mecanizadas y en donde se presume la desaparición de las leñosas dentro de la composición florística inicial (Eagler, 1954). Estas investigaciones deben considerar también el estudio de la vegetación que se desarrolla dentro de los helechales en estas condiciones, así como sus referentes ecológicos, como lo son los acahuales y la vegetación madura.

\section{CONCLUSIONES}

- La presencia prolongada del helecho restituye la fertilidad del suelo y restaura su biota, incluso a niveles superiores a los alcanzados por la condición de referencia, es decir la VM. Además, la incorporación de las frondas del helecho al suelo (mantillo) favorece un efecto estabilizador de la acidez que promueve una condición en donde podrían ser más viables las acciones de restauración/rehabilitación.

- La presencia prolongada del helecho no causa mayor impacto en la abundancia de macrofauna del suelo ni de los gremios de los detritívoros e ingenieros del suelo de las zonas invadidas.

- Tomando en cuenta las variables químicas y biológicas del suelo, los helechales cuentan con una mejor calidad de suelo en relación a la vegetación madura. Desde esta perspectiva no se tienen evidencias para considerar a los helechales como una condición degradada.

Cuadro 4. Valores medios de las abundancias de los gremios más representativos: detritívoros e ingenieros del suelo en helechales jóvenes (HJ), viejos (HV) y vegetación madura (VM), ubicados en el ejido Ing. Ricardo Payró Jene.

Table 4. Average values of the abundances of the most representative guilds: detritivores and soil engineers in young (HJ), old (HV) and mature vegetation (VM) ferns, located in the ejido Ing. Ricardo Payró Jene.

\begin{tabular}{lccccc}
\hline Variable & Condición & Media $^{\dagger}$ & Media cuadrática & F & P \\
\hline Detritívoros & VM & 16.5 & 0.66 & 2.26 & 0.15 \\
& $\mathrm{HV}$ & 10.7 & & & \\
Ingenieros del suelo & $\mathrm{HJ}$ & 41.0 & & 0.32 & \\
& $\mathrm{VM}$ & 16.5 & 0.19 & & \\
& $\mathrm{HV}$ & 35.5 & & & \\
& $\mathrm{HJ}$ & 6.2 & & & \\
\end{tabular}

$\overline{\mathrm{VM}}=$ vegetación madura; $\mathrm{HV}=$ helechales viejos; $\mathrm{HJ}=$ jóvenes. ${ }^{\dagger}$ Los valores de la media cuadrática corresponden a los valores transformados $(\log 10(\mathrm{x}+1))$, los valores medios no están transformados.

$\mathrm{VM}=$ mature vegetation; $\mathrm{HV}=$ old ferns; $\mathrm{HJ}=$ young. ${ }^{\dagger}$ The root mean square values correspond to the transformed values $(\log 10(\mathrm{x}+1))$, the mean values are not transformed. 
En particular, llama la atención los HV en los cuales, aparentemente, cuentan con pocos rizomas vivos y un alto porcentaje de rizomas muertos, comparado con el HJ. Estas dos circunstancias podrían dar lugar a suponer que los HV son propicios para su aprovechamiento agrícola o para llevar a cabo acciones de restauración o rehabilitación. Sin embargo, esta posibilidad se considera con reservas, dado que la presencia de los rizomas y la producción de las frondas de $P$. aquilinum es la principal restricción para la siembra de cultivos de estas áreas.

- Los resultados obtenidos hacen suponer que el "desuso" de las áreas invadidas por el helecho es consecuencia de la presencia y competencia que los helechales ejercen en los cultivos a través de sus rizomas, y no del deterioro de los atributos químicos y biológicos del suelo.

\section{AGRADECIMIENTOS}

Agradecemos al Sr. Santana de los Santos Sánchez y su familia por su valioso apoyo en el trabajo de campo. Esta investigación fue realizada como parte de una investigación de posgrado apoyada con una beca del Consejo Nacional de Ciencia y Tecnología. Se agradece el financiamiento por parte de U.S. Fish and Wildlife Service (Grant \# F16AP00239), del Fondo Mexicano para la Conservación, La dirección de la Reserva de la Biosfera de Calakmul, Etnobiología para la Conservación A.C. y el Fondo de Investigación Científica y Desarrollo Tecnológico de El Colegio de la Frontera Sur (FID 784) que hicieron posible el desarrollo de este trabajo.

De manera específica se agradecen los aportes de Perla Victoria Rodríguez y Gibrán Sánchez en la revisión del documento, así como a Filiberto Moisés por la elaboración del mapa.

\section{LITERATURA CITADA}

Adame-González, A. B. 2011. Micropropagación de Pteridium aquilinum (L.) Kuhn. Universidad Nacional Autónoma de México.

Anderson, J. M. and J. S. I. Ingram. 1993. Tropical soil biology and fertility: A handbook of methods. CABI Publishing. Wallingford, UK. ISBN-13: 978-0851988214.

Berget, C., E. Duran, and D. Barton-Bray. 2015. Participatory restoration of degraded agricultural areas invaded by bracken fern (Pteridium aquilinum) and conservation in the Chinantla Región, Oaxaca, Mexico. Hum. Ecol. 43: 547-558. doi: 10.1007/s10745-015-9762-0.
Brussaard, L. 1998. Soil fauna, guilds, functional groups and ecosystem processes. Appl. Soil Ecol. 9: 123-135. doi: https:// doi.org/10.1016/S0929-1393(98)00066-3.

Cabrera, G. 2012. La macrofauna edáfica como indicador biológico del estado de conservación/perturbación del suelo. Resultados obtenidos en Cuba. Pastos y Forrajes 35: 349-364.

CONABIO (Comisión Nacional para el Conocimiento y Uso de la Biodiversidad). 2013. La biodiversidad en Chiapas: Estudio de Estado. Comisión Nacional para el Conocimiento y Uso de la Biodiversidad/Gobierno del Estado de Chiapas. México. ISBN: 978-607-7607-98-4.

Corporation, I. 2012. Guía breve de IBM SPSS Statistics 21. IBM ${ }^{\circledR}$ SPSS ${ }^{\circledR}$ Statistics Versión 21.

Crane, M. 1990. Pteridium aquilinum. Eff. Inf. Syst. [Online]. U.S. Dep. Agric. For. Serv. Rocky Mt. Res. Station. Fire Sci. Lab. (Producer). http://www.fs.fed.us/database/feis/plants/fern/ pteaqu/all.html (Consulta: mayo 18, 2018).

DeLuca, T., S. A. Zewdie, O. Zackrisson, J. R. Healey, and D. L. Jones. 2013. Bracken fern (Pteridium aquilinum L . kuhn ) promotes an open nitrogen cycle in heathland soils. Plant Soil 367: 521-534. doi: https://doi.org/10.1007/s11104-012-14840.

Doi, R. and K. Sakurai. 2004. Principal components derived from soil physico-chemical data explained a land degradation gradient, and suggested the applicability of new indexes for estimation of soil productivity in the Sakaerat Environmental Research Station, Thailand. Int. J. Sust. Dev. World Ecol. 11: 298-311. doi: https://doi.org/10.1080/13504500409469833.

Eagler, F. E. 1954. Vegetation science concepts, I. Initial floristic composition, a factor in old field vegetation development. Vegetatio 14: 412-417. doi: https://doi.org/10.1007/ BF00275587.

FAO (Organización de las Naciones Unidas para la Alimentación y la Agricultura). 2015. World Reference Base for Soil Resources 2014, update 2015. World Soil Resources Reports No. 106. FAO, Rome.

Gálvez, A., A. Reina y E. Meneses. 2016. Cuantificación de macrofauna edáfica en un sistema silvopastoril y uno convencional en bosque seco. Rev. Investig. Pecu. 4: 13-25.

García, E. 2004. Modificaciones al sistema de clasificación climática de Köppen. Instituto de Geografía, UNAM. México, D. F., México.

Ghorbani, J., P. M. Das, A. B. Das, J. M. Hughes, H. A. McAllister, S. K. Pallai, R. J. Pakeman, R. H. Marrs, and M. G. Le Duc. 2003. Effects of restoration treatments on the diaspore bank under dense Pteridium stands in the UK. Appl. Veg. Sci. 6: 189-198. doi: https://doi.org/10.1111/j.1654-109X.2003. tb00579.x.

Ghorbani, J., M. G. Le Duc, H. A. McAllister, R. J. Pakeman, and R. H. Marrs. 2006. Effects of the litter layer of Pteridium aquilinum on seed banks under experimental restoration. Appl. Veg. Sci. 9: 127-136. doi: https://doi.org/10.1111/j.1654109X.2006.tb00662.x.

Green, D. N. and M. A. Nishimura. 2011. Forest succession and restoration in Imperata cylindrica- and fern-dominated communities in Tanjung Puting National Park. Indonesia. Proquest, Umi Dissertation Publishing. University of California, Los Angeles. ISBN-13: 978-1243418906.

Hernández-Vargas, G., L. R. Sánchez-Velásquez, J. C. LópezAcosta, J. C. Noa-Carrazana, and Y. Perroni. 2019. 
Relationship between soil properties and leaf functional traits in early secondary succession of tropical montane cloud forest. Ecol. Res. 34: 213-224. doi: https://doi.org/10.1111/14401703.1267.

He, X., Y. Chen, S. Liu, A. Gunina, X. Wang, W. Chen, S. Yuanhu, L. Shi, Q. Yao, J. Li, X. Zou, J. P. Schimel, W. Zhang, and S. Fu. 2018. Cooperation of earthworm and arbuscular mycorrhizae enhanced plant $\mathrm{N}$ uptake by balancing absorption and supply of ammonia. Soil Biol. Biochem. 116: 351-359. doi: https:// doi.org/10.1016/j.soilbio.2017.10.038.

Huerta, E., J. Rodríguez-Olán, I. Evia-Castillo, E. MontejoMeneses, M. De la Cruz-Mondragón y R. García-Hernández. 2008. Relación entre la fertilidad del suelo y su población de macroinvertebrados. Terra Latinoamericana 26: 171-181.

INEGI (Instituto Nacional de Estadística y Geografía). 2009. Prontuario de información geográfica municipal de los Estados Unidos Mexicanos Calakmul, Campeche. Clave geoestadística 04010. México, D. F.

Jones, C. G. and R. D. Firn. 1979. Some allelochemicals of Pteridium aquilinum and their involvement in resistance to Pieris brassicae. Biochem. Syst. Ecol. 7: 187-192. doi: https:// doi.org/10.1016/0305-1978(79)90048-6.

Kloster, N., M. Pérez y A. Bono. 2016. Análisis del carbono total, orgánico e inorgánico en suelos de la Región Semiárida Pampeana Argentina. Cienc. Suelo 34: 365-372.

Levy-Tacher, S. I., I. Vleut, F. Román, and J. Aronson. 2015. Natural regeneration after long-term bracken fern control with balsa (Ochroma pyramidale) in the neotropics. Forests 6 : 2163-2177. doi: 10.3390/f6062163.

Marrs, R. H. and A. S. Watt. 2006. Biological flora of the British Isles: Pteridium aquilinum (L.) Kuhn. J. Ecol. 94: 1272-1321. doi: 10.1111/j.1365-2745.2006.01177.x.

Martínez, E., J. Fuentes y E. Acevedo. 2008. Carbono orgánico y propiedades del suelo. R. C. Suelo Nutr. Veg. 8: 68-96. doi: http://dx.doi.org/10.4067/S0718-27912008000100006.

Milligan, G., K. E. Booth, E. S. Cox, R. J. Pakeman, M. Le Duc, L. Connor, S. Blackbird, and R. M. Marrs. 2018. Change to ecosystem properties through changing the dominant species: Impact of Pteridium aquilinum control and heathland restoration treatments on selected soil properties. J. Environ. Manage. 207: 1-9. doi: https://doi.org/10.1016/j. jenvman.2017.11.013.

Miranda, F. y E. Hernández. 1963. Los tipos de vegetación de México y su clasificación. Bot. Sci. 28: 29-179. doi: https:// doi.org/10.17129/botsci.1084.

Morón, A. and E. Huerta. 2006. Soil macrofauna of two successional evergreen cloud forest stages from de Cerro Huitepec Nature Reserve, San Cristóbal de Las Casas, Chiapas, México. Interciencia 31: 611-615.
Ortega M., F. J. 1989. La etnobotánica de Pteridium aquilinum (L.) Kuhn en Venezuela y sus posibles riesgos asociados de carcinogénesis. Medula 2: 51-56.

Pakeman, R. J., R. H. Thwaites, M. G. Le Duc, and R. H. Marrs. 2000. Vegetation re-establishment on land previously subject to control of Pteridium aquilinum by herbicide. Appl. Veg. Sci. 3: 95-104. doi: https://doi.org/10.2307/1478923.

Pakeman, R. J., R. H. Thwaites, M. G. Le Duc, and R. H. Marrs. 2002. The effects of cutting and herbicide treatment on Pteridium aquilinum encroachment. Appl. Veg. Sci. 5: 203212. doi: https://doi.org/10.1111/j.1654-109X.2002.tb00550.x.

Ramírez Trejo, M. R., B. Pérez García, D. R. Pérez Salicrup, and A. Orozco Segovia. 2010. Effect of fire on the germination of spores of Pteridium caudatum, an invasive fern. J. Trop. Ecol. 26: 457-465. doi: https://www.jstor.org/stable/40665283.

Rasmussen, L. H., S. Kroghsbo, J. C. Frisvad, and H. C. Hansen. 2003. Occurrence of the carcinogenic Bracken constituent ptaquiloside in fronds, topsoils and organic soil layers in Denmark. Chemosphere 51: 117-127. doi: 10.1016/s00456535(02)00694-x.

Samecka, A., K. Garbiec, K. Kolon, and A. Kempers. 2009. Factor analysis of the elemental composition of Pteridium aquilinum from serpentine and granite soils as a tool in the classification of relations between this composition and the type of parent rock in the Sleza Massif in Lower Silesia, Poland. Environ. Geol. 58: 509-514. doi: 10.1007/s00254-008-1524-5.

Schneider, L. 2004a. Bracken fern invasion in Southern Yucatán: a case for Land-Change Science. Geogr. Rev. 94: 229-241.

Schneider, L. 2006. Invasive species and land-use : The effect of land management practices on bracken fern invasion in the region of Calakmul, Mexico. J. Latin Am. Geogr. 5: 92-107. doi: 10.1353/lag.2006.0028.

SEMARNAP (Secretaria del Medio Ambiente, Recursos Naturales y Pesca). 1999. Programa de manejo de la Reserva de la Biosfera de Calakmul. 1a edición. Instituto Nacional de Ecología. México D. F.

SEMARNAT (Secretaría de Medio Ambiente y Recursos Naturales). 2002. Norma Oficial Mexicana NOM-021-SEMARNAT-2000 que establece las especificaciones de fertilidad, salinidad y clasificación de suelos, estudio, muestreo y análisis. SEMARNAT. México, D. F.

Suazo, I., L. Lopez, J. Alvarado, and M. Martínez. 2015. Landuse change dynamics, soil type and species forming monodominant patches: the case of Pteridium aquilinum in a neotropical rain forest region. Biotropica 47: 18-26. doi: https://doi.org/10.1111/btp.12181.

Velasquez, E., P. Lavelle, and M. Andrade. 2007. GISQ, a multifunctional indicator of soil quality. Soil Biol. Biochem. 39: 3066-3080. doi: 10.1016/j.soilbio.2007.06.013. 